\title{
Atividade laboral em pacientes atendidos em um serviço ambulatorial de dor crônica*
}

\section{Labor activity for patients seen in a chronic pain outpatient setting}

João Batista Santos Garcia', Elmar Torres Neto²

* Recebido do Hospital Universitário da Universidade Federal do Maranhão (UFMA). São Luis, MA.

\section{RESUMO}

JUSTIFICATIVA E OBJETIVOS: O presente estudo objetivou determinar o perfil clínico dos pacientes em atividade remunerada e licença-saúde atendidos no serviço de dor crônica, envolvendo características sociodemográficas, intensidade da dor, principais alterações do exame físico e terapêutica proposta para cada caso.

MÉTODO: Trata-se de um estudo transversal no qual foram analisados retrospectivamente prontuários de pacientes atendidos no serviço ambulatorial de dor crônica do Hospital Universitário da Universidade Federal do Maranhão (HU-UFMA). Um total de 308 pacientes foi avaliado através de informações que foram obtidas por meio de fichas-protocolo já existentes no serviço, que continham dados referentes aos objetivos da pesquisa.

RESULTADOS: O sexo feminino foi maior no grupo em atividade $52,5 \%$ e o masculino maior no grupo em licença $65,5 \%(\mathrm{p}=0,028)$. A média de idade dos pacientes foi de 49,34 $\pm 14,01$ anos. Os principais diagnósticos foram lombalgia com $100(32,61 \%)$ pacientes, seguida de osteoartrose com $23(7,76 \%)$ pacientes. A intensidade da dor crônica não apresentou

1. Professor Adjunto Doutor da Disciplina de Anestesiologia, Dor e Cuidados Paliativos da Universidade Federal do Maranhão (UFMA). Responsável pelo Serviço de Dor do Hospital Universitário UFMA e do Instituto Maranhense de Oncologia Aldenora Belo. São Luis, MA, Brasil.

2. Graduando do Curso de Medicina da Universidade Federal do Maranhão (UFMA). Membro da Liga Acadêmica de Dor do Maranhão (LAD). São Luis, MA, Brasil.

Endereço para correspondência:

Dr. João Batista Santos Garcia

Av. São Marcos, L 4, Q C, Apt 502

65077-310 São Luís, MA.

Fone: (98) 3227-4508

E-mail: jbgarcia@uol.com.br diferença significante entre os dois grupos analisados $(48,6 \%$ versus $60,9 \%)$, assim como não foram observadas alteração da força, movimento e sono entre os dois grupos. A fidelidade ao tratamento foi maior no grupo em atividade $(86,4 \%$ versus $64,3 \%)$ $(\mathrm{p}=0,028)$ e o grupo de fármaco mais frequentemente utilizado foi o analgésico comum em ambos os grupos.

CONCLUSÃO: Os pacientes que estavam em licença-saúde foram predominantemente homens com intensidade dolorosa e alterações do exame físico semelhantes aos que estavam em atividade remunerada, entretanto, com menor aderência ao tratamento proposto.

Descritores: Atividade laboral, Dor crônica, Licença-saúde.

\section{SUMMARY}

BACKGROUND AND OBJECTIVES: This study aims at determining the clinical profile of patients in paid activity or medical leave seen in the chronic pain service, involving socio-demographic characteristics, pain intensity, major physical evaluation changes and therapy proposed to each case.

METHOD: This is a transversal study which has retrospectively analyzed the medical charts of patients seen by the chronic pain outpatient setting of the University Hospital, Federal University of Maranhão (HU-UFMA). A total of 308 patients were evaluated through information obtained by protocol-records existing in the service, and which contained data related to the objectives of this research.

RESULTS: There has been predominance of females in the activity group (52.5\%) and of males in the medical leave group $(65.5 \%)(\mathrm{p}=0.028)$. Mean age of patients was $49.34 \pm 14.01$ years. Primary diagnoses were low back pain in 100 patients $(32.61 \%)$, followed by osteoarthritis in 23 patients $(7.76 \%)$. Chronic 
pain intensity was not significantly different between groups (48.6\% vs. $60.9 \%)$ and strength, movement and sleep changes were not observed in both groups. Loyalty to treatment was higher in the active group (86.4\% vs.64.3\%) and analgesics were the most common drugs used by both groups.

CONCLUSION: Patients in medical leave were predominantly males with pain intensity and physical evaluation changes similar to those in activities, however with lower adherence to treatment.

Keywords: Chronic pain, Labor activity, Medical leave.

\section{INTRODUÇÃO}

A dor crônica, atualmente, é considerada um problema de saúde pública ${ }^{1}$. Estudos epidemiológicos relatam taxas abrangentes que são, muitas vezes, devido às diferentes definições de dor crônica e às diferentes metodologias utilizadas. Em uma revisão de estudos epidemiológicos realizados em diferentes partes do mundo, as taxas de prevalência de dor crônica variaram entre $12 \%$ e $80 \%{ }^{2}$. Estudo realizado no Brasil na população de Salvador mostrou prevalência de $41,4 \%{ }^{3}$. Outro estudo, realizado somente com idosos em Londrina, mostrou prevalência de $51,4 \%{ }^{4}$. Recentemente em estudo realizado na cidade de São Luís encontrou-se a prevalência de $42 \%$, sendo $10 \%$ dor neuropática 5 .

Em virtude da dor, grande parte dos pacientes se torna parcial ou totalmente incapacitados, transitória ou permanentemente ${ }^{6}$, desencadeando acentuado estresse, sofrimento e perda na qualidade de vida, sendo considerada a maior causa de absenteísmo do trabalho, gerando ônus para a sociedade ${ }^{7}$.

A dor lombar, por exemplo, é um problema de alto custo médico e social nos Estados Unidos, sendo causa de perda de 1.400 dias de trabalho por mil habitantes por ano; na Europa, é a mais frequente causa de limitação em pessoas com menos de 45 anos e a segunda causa mais frequente de consulta médica ${ }^{8}$. Na Holanda, são registrados 10.000 casos novos, a cada ano, de pacientes incapacitados para o trabalho pela dor'.

Dessa forma, o absenteísmo traz, nos diversos países, independentemente do seu grau de desenvolvimento, um aumento direto dos custos pela concessão de auxílio-doença, benefício concedido ao segurado impedido de trabalhar por doença ou acidente por mais de 15 dias consecutivos, e um aumento indireto nos custos pela diminuição da produtividade e da eficiência, e ainda maior desperdício e aumento dos problemas administrativos com sucessivas substituições dos trabalhadores faltosos ${ }^{10}$.
Em 2009, a Previdência Social do Brasil concedeu 4,5 milhões de benefícios, dos quais $83,9 \%$ eram previdenciários. A espécie mais concedida foi o auxílio-doença, correspondendo a $38,3 \%$ do total. O valor total dos benefícios concedidos em 2009 atingiu R\$ 3,18 bilhões. Considerando o valor dos benefícios, as espécies mais concedidas foram todas previdenciárias: o auxílio-doença, a aposentadoria por tempo de contribuição e a aposentadoria por idade, cujas participações foram de $41,5 \%, 10,8 \%$ e $10,3 \%$, respectivamente ${ }^{11,12}$.

O estudo sobre o absenteísmo nas atividades laborais de pacientes com dor crônica é fundamental para o desenvolvimento de medidas de prevenção e tratamento adequados, importante para a melhoria na qualidade de vida do paciente. A redução do número de dias de trabalho perdidos, resultado das incapacidades geradas pela dor crônica, também é útil para diminuir o impacto desse problema sobra a Previdência Social ${ }^{11,12}$.

O objetivo deste estudo foi determinar o perfil clínico dos pacientes em atividade remunerada e os em licença-saúde atendidos no serviço de dor crônica, envolvendo características sociodemográficas, intensidade da dor, principais alterações do exame físico e terapêutica proposta para cada caso, bem como a escassez de dados epidemiológicos sobre esse tema, abordando esse aspecto da dor crônica.

\section{MÉTODO}

Após aprovação pelo Comitê de Ética em Pesquisa do Hospital Universitário da Universidade Federal do Maranhão (HU-UFMA) (processo $n^{0} 33104$ 1222/2007), realizou-se este estudo transversal, retrospectivo, descritivo em que foram analisados os dados demográficos, história clínica, exame físico e a terapêutica instituída em pacientes com dor crônica atendidos no período de 2006 a 2010, no serviço ambulatorial de dor crônica.

Os dados foram coletados a partir de fichas de primeira consulta com questionamentos pré-determinados, padronizados e respaldados pelo serviço de dor. Com base nas informações fornecidas pelos pacientes e registradas nas fichas foi feita uma caracterização pormenorizada da dor. A intensidade do quadro doloroso foi classificada, de acordo com a escala verbal, em leve, moderada e intensa. O tempo de início da dor foi registrado em meses e a sua localização foi indicada pelo paciente. Quanto às limitações provocadas pela dor, 
avaliou-se a alteração da força muscular, de acordo com os critérios da Medical Research Council (MRC) em que grau $0=$ plegia ausência de tônus muscular, grau 1 = tônus muscular presente, grau $2=$ movimentação no plano, grau 3 = vence a gravidade sem resistência, grau $4=$ vence a gravidade contra resistência e grau 5 = força muscular normal; além de alteração do movimento e do sono, de acordo com informações referidas pelo paciente. Os dados sobre fidelidade ao tratamento também foram baseados em relatos do paciente, sendo considerados fiéis apenas aqueles que seguiram rigorosamente a terapêutica instituída.

Foi criado um banco de dados utilizando-se o software Microsoft Office Excel 2007. A análise estatística foi realizada através do software Epi Info 3.5.1. Os resultados foram expressos por meio de frequências com porcentagem e médias \pm desvios-padrão e medianas. A regressão logística foi utilizada para verificar a diferença na prevalência entre os grupos cuja variável dependente do estudo foi a presença de licença-saúde. Em relação às questões específicas para dor foi aplicado o teste do Qui-quadrado, além dos testes $t$ de Student e Mann-Whitney para as variáveis com médias e medianas. Considerou-se valor de $\mathrm{p}<0,05$ como estatisticamente significativo.

\section{RESULTADOS}

Foram incluídos 308 pacientes no período estudado e houve um grande número de exclusão devido a falhas no preenchimento das fichas, tornando os dados incompletos.

Dos 308 pacientes atendidos, 45,5\% eram do sexo masculino e $54,5 \%$ do sexo feminino. A média de idade foi de 49,34 anos $\pm 14,01$. A maioria $(43,3 \%)$ concluiu o ensino fundamental e apenas $11,9 \%$ tinha concluído o ensino superior. Mais de 50\% dos pacientes eram casados e $27,5 \%$ eram solteiros. Cerca de $60 \%$ dos pacientes eram provenientes da capital, enquanto $38,6 \%$ eram provenientes do interior do estado. Metade dos pacientes tinha renda de até um salário mínimo e apenas 5,5\% apresentavam renda superior a cinco salários mínimos. Os principais diagnósticos foram lombalgia $(32,6 \%)$, seguido de osteoartrose $(7,7 \%)$, entre outros como cefaleia, fibromialgia e dor neuropática. Pela escala verbal, a dor foi referida como intensa em 47,2\% dos pacientes. Cerca de $40 \%$ dos pacientes exerciam atividade remunerada e $21,3 \%$ estavam em licença-saúde (Tabela 1).
Tabela 1 - Características sociodemográficas e clínicas dos pacientes.

\begin{tabular}{|c|c|}
\hline Características & Pacientes $(\mathrm{n}=308)$ \\
\hline Idade (anos, média \pm DP) & $49,34 \pm 14,01$ \\
\hline \multicolumn{2}{|l|}{ Sexo } \\
\hline Masculino & $45,5 \%$ \\
\hline Feminino & $54,5 \%$ \\
\hline \multicolumn{2}{|l|}{ Localização } \\
\hline Lombalgia & $32,61 \%$ \\
\hline Osteoartrose & $7,76 \%$ \\
\hline Outros & $59,7 \%$ \\
\hline \multicolumn{2}{|l|}{ Estado conjugal } \\
\hline Casado & $58,7 \%$ \\
\hline Solteiro & $27,5 \%$ \\
\hline Viúvo & $6,1 \%$ \\
\hline Divorciado & $7,7 \%$ \\
\hline \multicolumn{2}{|l|}{ Profissão } \\
\hline Atividade remunerada & $38,4 \%$ \\
\hline Atividade sem remuneração & $8,1 \%$ \\
\hline Sem atividade & $15,5 \%$ \\
\hline Aposentado & $16,7 \%$ \\
\hline Licença-saúde & $21,3 \%$ \\
\hline \multicolumn{2}{|l|}{ Procedência } \\
\hline Capital & $61 \%$ \\
\hline Interior & $38,6 \%$ \\
\hline Outros & $0,4 \%$ \\
\hline \multicolumn{2}{|l|}{ Escolaridade } \\
\hline Analfabeto & $10,4 \%$ \\
\hline Ensino fundamental & $43,3 \%$ \\
\hline Ensino médio & $34,3 \%$ \\
\hline Ensino superior & $11,9 \%$ \\
\hline \multicolumn{2}{|l|}{ Renda familiar } \\
\hline Até $1 \mathrm{SM}$ & $50 \%$ \\
\hline Até $3 \mathrm{SM}$ & $36 \%$ \\
\hline Até 5 SM & $8,5 \%$ \\
\hline Acima de 5 SM & $5,5 \%$ \\
\hline \multicolumn{2}{|l|}{ Escala verbal de dor } \\
\hline Leve & $9,2 \%$ \\
\hline Moderada & $43,6 \%$ \\
\hline Intensa & $47,2 \%$ \\
\hline
\end{tabular}

$\mathrm{SM}=$ salário(s) mínimo(s); DP = desvio-padrão

Analisando separadamente os 99 pacientes que exerciam atividade remunerada, observou-se que 52,5\% eram do sexo feminino e $47,5 \%$ do sexo masculino ( $p$ 
$=0,028)$. A média de idade desses pacientes foi de 45 anos, variando de 23 a 77 anos. Nesse grupo, 58,1\% eram casados e $29,1 \%$ solteiros. A maioria dos pacientes era proveniente da capital $(66,3 \%)$, tinham ensino médio completo $(46,4 \%)$ e ganhavam até três salários mínimos (42,9\%). A mediana do tempo de início da dor nesses pacientes foi de $36 \pm 12,6$ meses, sendo considerada leve em $8,2 \%$ e intensa em $48,6 \%$ deles, de acordo com a escala verbal (Tabela 2).

Houve alteração do sono e do movimento em $64,9 \%$ e em $79 \%$ dos pacientes, respectivamente. Apenas $34,5 \%$ dos pacientes apresentaram alteração da força muscular. Com relação ao tratamento farmacológico para a dor, observou-se uma predominância do uso de analgésicos não opioides (37,3\%), com uma fidelidade ao tratamento de $86,4 \%(\mathrm{p}=0,028)$ (Tabela 3$)$.

Tabela 2 - Comparação das características sociodemográficas dos pacientes em atividade remunerada e licença-saúde.

\begin{tabular}{|c|c|c|c|}
\hline \multirow{2}{*}{ Variáveis } & \multicolumn{2}{|c|}{ Licença-Saúde } & \multirow{2}{*}{$\begin{array}{l}\text { Valor } \\
\text { de } p\end{array}$} \\
\hline & Sim & Não & \\
\hline Idade (anos, média \pm DP) & $46 \pm 10,76$ & $45,2 \pm 9,37$ & $0,634 * *$ \\
\hline \multicolumn{4}{|l|}{ Sexo } \\
\hline Masculino & $65,5 \%$ & $47 \%$ & \multirow[t]{2}{*}{0,028} \\
\hline Feminino & $34,5 \%$ & $53 \%$ & \\
\hline \multicolumn{4}{|l|}{ Estado Civil } \\
\hline Casado & $58 \%$ & $58,1 \%$ & \multirow{4}{*}{0,711} \\
\hline Divorciado & $8 \%$ & $10,5 \%$ & \\
\hline Solteiro & $28 \%$ & $29,1 \%$ & \\
\hline Viúvo & $6 \%$ & $2,3 \%$ & \\
\hline \multicolumn{4}{|l|}{ Procedência } \\
\hline Capital & $59 \%$ & $63,3 \%$ & \multirow[t]{2}{*}{0,292} \\
\hline Interior & $38,6 \%$ & $33,7 \%$ & \\
\hline \multicolumn{4}{|l|}{ Escolaridade } \\
\hline Analfabeto & $4,9 \%$ & $7,2 \%$ & \multirow{4}{*}{0,066} \\
\hline Ensino fundamental & $48,8 \%$ & $26,1 \%$ & \\
\hline Ensino médio & $39 \%$ & $46,4 \%$ & \\
\hline Ensino superior & $7,3 \%$ & $20,3 \%$ & \\
\hline \multicolumn{4}{|l|}{ Renda } \\
\hline Até $1 \mathrm{SM}$ & $59,5 \%$ & $41,1 \%$ & \multirow{4}{*}{0,363} \\
\hline Até $3 \mathrm{SM}$ & $27 \%$ & $42,9 \%$ & \\
\hline Até $5 \mathrm{SM}$ & $10,8 \%$ & $12,5 \%$ & \\
\hline Acima de $5 \mathrm{SM}$ & $2,7 \%$ & $3,6 \%$ & \\
\hline
\end{tabular}

Porcentagens comparadas pela regressão logística.

** Médias comparadas pelo teste $\mathrm{t}$ de Student. $\mathrm{SM}=$ salário mínimo; DP = desvio-padrão.
Tabela 3 - Comparação das características clínicas da dor dos pacientes em atividade remunerada e licença-saúde.

\begin{tabular}{|c|c|c|c|}
\hline \multirow{2}{*}{ Variáveis } & \multicolumn{2}{|c|}{ Licença-Saúde } & \multirow{2}{*}{$\begin{array}{l}\text { Valor } \\
\text { de } p\end{array}$} \\
\hline & Sim & Não & \\
\hline $\begin{array}{l}\text { Tempo de dor } \\
\text { (em meses) }\end{array}$ & $36(12 ; 60)^{*}$ & $36(12 ; 60)^{*}$ & $0,990 \dagger$ \\
\hline \multicolumn{4}{|l|}{ Escala verbal } \\
\hline Leve & $6,5 \%$ & $8,2 \%$ & \multirow{3}{*}{0,465} \\
\hline Moderada & $32,6 \%$ & $43,2 \%$ & \\
\hline Intensa & $60,9 \%$ & $48,6 \%$ & \\
\hline
\end{tabular}

Alteração do

sono

$\begin{array}{lll}\text { Sim } & 72,7 \% & 64,9 \% \\ \text { Não } & 27,3 \% & 35,1 \%\end{array}$

Alteração do

movimento

\begin{tabular}{|c|c|c|c|}
\hline & \multirow{3}{*}{0,944} \\
\hline Sim & $79,5 \%$ & $79 \%$ & \\
\hline Não & $20,5 \%$ & $21 \%$ & \\
\hline \multicolumn{4}{|c|}{ Alteração de força } \\
\hline Grau 0 & $7 \%$ & $0 \%$ & \multirow{6}{*}{0,107} \\
\hline Grau 1 & $0 \%$ & $0 \%$ & \\
\hline Grau 2 & $0 \%$ & $0 \%$ & \\
\hline Grau 3 & $11,6 \%$ & $7,1 \%$ & \\
\hline Grau 4 & $23,3 \%$ & $27,4 \%$ & \\
\hline Grau 5 & $58,1 \%$ & $65,5 \%$ & \\
\hline \multicolumn{4}{|l|}{$\begin{array}{l}\text { Tratamento } \\
\text { farmacológico }\end{array}$} \\
\hline Nenhum & $2 \%$ & $2 \%$ & \multirow{6}{*}{0,441} \\
\hline $\begin{array}{l}\text { Analgésicos } \\
\text { não opioides }\end{array}$ & $33,3 \%$ & $37,3 \%$ & \\
\hline Opioide fraco & $19,4 \%$ & $17,6 \%$ & \\
\hline Opioide forte & $2,8 \%$ & $0 \%$ & \\
\hline Tricíclicos & $11,1 \%$ & $5,9 \%$ & \\
\hline Outros & $33,3 \%$ & $37,3 \%$ & \\
\hline \multicolumn{3}{|l|}{$\begin{array}{l}\text { Fidelidade } \\
\text { ao tratamento }\end{array}$} & \multirow{3}{*}{0,028} \\
\hline $\operatorname{Sim}$ & $64,3 \%$ & $86,4 \%$ & \\
\hline Não & $35,7 \%$ & $13,6 \%$ & \\
\hline
\end{tabular}

* Mediana (p25; p75). Porcentagens comparadas pela análise pelo teste Qui-quadrado. ** Medianas comparadas pelo teste de Mann-Whitney.

O grupo em licença médica correspondeu a 55 pacientes, dentre os quais $65,5 \%$ eram do sexo masculino e $34,5 \%$ do sexo feminino $(\mathrm{p}=0,028)$. A média de idade dos pacientes foi de 46 anos, com idade mínima de 20 anos e máxima de 73 anos. A maioria dos pacientes era casada 
(58\%) e $59,1 \%$ provenientes da capital. Quase metade dos pacientes $(48,8 \%)$ tinha apenas o ensino médio completo e 7,3\% possuía ensino superior completo. A renda foi de até um salário mínimo para 59,5\% dos pacientes (Tabela 2). A mediana do tempo de início da dor foi de $36 \pm 12,6$ meses. A dor foi considerada intensa por $60,9 \%$ dos pacientes, de acordo com a escala verbal de dor.

A alteração do sono foi observada em $72,7 \%$ dos pacientes, 79,5\% referiram alteração do movimento e 41,9\% apresentavam algum tipo de alteração da força (Tabela 3). Os analgésicos não opioides foram o principal fármaco utilizado para o tratamento da dor crônica, representando $33,3 \%$. A maioria dos pacientes $(64,3 \%)$ afirmou ser fiel ao tratamento $(\mathrm{p}=0,028)$ (Tabela 3$)$.

\section{DISCUSSÃO}

A característica incapacitante da dor foi evidenciada em grande quantidade de pacientes em licença-saúde $(21,3 \%)$. Estudo realizado no Reino Unido ${ }^{13}$ também encontrou relação entre dor crônica e indivíduos afastados e incapacitados, comparado com indivíduos empregados. Da mesma forma, outros autore ${ }^{14}$ constataram, em estudo abrangente, que dentre os pacientes com dor crônica e, consequente comprometimento da atividade profissional, 8,4\% afirmaram já ter faltado ao trabalho devido à dor e $22,3 \%$ disseram que abandonaram o emprego em consequência dela.

A média de idade nos grupos em atividade remunerada e em licença-saúde foi 45 e 46 anos, respectivamente. Os valores são semelhantes aos encontrados em estudo de dor lombar crônica em população adulta no sul do Brasil, no qual a média de idade foi $44 \pm 16,3 \operatorname{anos}^{14}$. A faixa etária afetada pela dor crônica gera interesse, pois os indivíduos em licença-saúde são trabalhadores ainda em idade produtiva ${ }^{15} \mathrm{e}$, consequentemente, a falta ao trabalho gera inúmeros transtornos organizacionais e econômicos.

A prevalência de dor crônica foi maior no sexo feminino, considerando o total de pacientes estudados $(54,5 \%)$ e aqueles do grupo em atividade remunerada analisados separadamente (52,5\%). Esses dados se assemelham ao estudo realizado na Holanda ${ }^{16}$, que demonstrou predominância de dor crônica em mulheres $(53,9 \%)$ e ao um estudo envolvendo a população da cidade de Salvador ${ }^{3}$, no qual $55,4 \%$ dos indivíduos com dor crônica eram do sexo feminino. No entanto, analisando o grupo em licença-saúde, observou-se que a dor crônica foi mais frequente nos homens $(65,5 \%)$. Esse fato poderia ser explicado pelo tipo de trabalho exercido pelos homens, que geralmente demanda maior esforço físico, tornando-os mais suscetíveis aos efeitos deletérios da dor crônica. Essa ideia é compartilhada por Waddell ${ }^{17}$ que afirma que o absenteísmo por lombalgia está mais relacionado às diferenças sociais e à organização do trabalho do que às diferenças biológicas entre homens e mulheres.

$\mathrm{O}$ estado civil foi outro fator em concordância com alguns outros estudos publicados ${ }^{3,18}$ nos quais os casados correspondem a um maior percentual dentre os indivíduos com dor crônica. Isto pode ter uma possível explicação baseada no fato de que para algumas pessoas a vida conjugal impõe obrigações e encargos que podem contribuir para potencializar o quadro álgico.

A escolaridade e a renda por terem íntima relação de causa e consequência merecem uma análise em conjunto. Os resultados seguiram a lógica já esperada com um predomínio do ensino médio e ganho mensal de até três salários mínimos no grupo em atividade remunerada, enquanto o grupo em licença-saúde apresentou maioria com apenas o ensino fundamental e ganho de até um salário mínimo por mês. Outro ponto interessante, é que o número de pessoas com ensino superior em atividade remunerada correspondeu aproximadamente ao triplo dos pacientes em licença-saúde (20,3\% versus $7,3 \%)$.

Tal fato pode ser explicado pelo tipo de atividade laboral desenvolvida por esses pacientes. Os trabalhos, que exigem menor qualificação profissional, e podem estar relacionadas a sobrecarga física, são afetados de forma mais expressiva pela dor crônica do que as atividades mais qualificadas ${ }^{19}$. Por outro lado, esses dados também podem refletir a falta de interesse dos pacientes em licença-saúde em continuar trabalhando, uma vez que não teriam perdas ao se aposentar devido aos salários já baixos.

A diferença entre os pacientes que relataram dor de forte intensidade foi inferior ao que se esperava naqueles que trabalhavam em relação ao grupo em licença-saúde (48,6\% versus $60,9 \%$ ). Esses dados demonstram que a intensidade da dor crônica em si não seria suficiente para definir a licença-saúde desses pacientes. Outras variáveis, como alterações do sono, do movimento e da força, ratificam essa afirmação, pois apresentam frequências similares em ambos os grupos.

No tratamento farmacológico, os analgésicos não opioides aparecem nos dois grupos como a classe mais utilizada. Esse fato é surpreendente, pois é de se esperar que pessoas consideradas incapacitadas para exercer seu trabalho deveriam fazer uso de classes diferentes de medicações daqueles que estão em plena atividade. Será que um analgésico comum pode resolver o problema dessas 
pessoas ou elas se dizem satisfeitas, pois na verdade a dor não é tão incapacitante assim?

Pensando nisso, foi pesquisada a fidelidade ao tratamento desses pacientes, pois este dado seria um indicativo da veracidade dos fatos relatados ao médico, já que é de se esperar que um paciente com mais dor se empenhe mais no seu tratamento. O resultado mais uma vez foi na contramão do que se esperava, pois o grupo dos pacientes em atividade remunerada apresentou um índice maior de fidelidade ao tratamento (86,4\% versus $64,3 \%$ ) gerando questionamentos. Até que ponto a dor realmente incomoda esses pacientes? Que tipo de ganho a dor pode lhes oferecer?

O presente estudo mostrou a dimensão do problema que envolve o absenteísmo e a dor crônica. Entretanto, destaca-se uma limitação do estudo que teve como base apenas a ficha de primeiro atendimento, fato que impossibilitou o seguimento dos pacientes e a extração de informações como, por exemplo, o retorno a atividade laboral. Dessa forma, espera-se que estes dados possam contribuir para uma reflexão mais profunda no que diz respeito ao tema e estimule novos estudos abordando de forma específica a dor crônica, o número de licenças e o número de dias de trabalho perdidos, já que a maioria dos estudos refere-se ao absenteísmo e auxílio-doença de maneira geral, incluindo diversas causas diferentes da dor. Os critérios utilizados para a concessão dos benefícios pela Previdência Social talvez precisem ser reanalisados a fim de evitar licenças desnecessárias e aumento dos gastos, principalmente no setor público.

\section{CONCLUSÃO}

Os pacientes que estavam em licença-saúde foram predominantemente homens com intensidade dolorosa e alterações do exame físico semelhantes aos que estavam em atividade remunerada, entretanto, com menor adesão ao tratamento proposto.

\section{REFERÊNCIAS}

1. Von Korff M, Dworkin SF, Le Resche L. Graded chronic pain status: an epidemiologic evaluation. Pain 1990;40(3):279-91.

2. Abu-Saad, Huijer H. Chronic pain: a review. J Med Liban 2010;58(1):21-7.

3. Sá KN, Baptista AF, Matos MA, Lessa I. Chronic pain and gender in Salvador population, Brazil. Pain 2008;139(3):498-506.

4. Dellaroza MS, Pimenta CA, Matsuo T. Prevalence and characterization of chronic pain among the elderly living in the community. Cad Saude Publica 2007;23(5):1151-60.
5. Moraes VE, Garcia JB, Silva AA, et al. Prevalence, characteristics and factors associated with chronic pain with and without neuropathic characteristics in São Luís, Brazil. J Pain Symp Manag 2011; in press.

6. Breivik H, Collet B, Ventafrida V, et al. Survey of chronic pain in Europe: prevalence, impact on daily life and treatment. Eur J Pain 2006;10(4):187-333.

7. Blyth F. Chronic pain- is it a public health problem? Pain 2008;137(3):465-6.

8. Cavanaugh JM, Weinstein JN. Low back pain: epidemiology, anatomy and neurophysiology. In: Wall PD, Melzack R, (organizadores). Textbook of pain. New York (NY): Livingstone; 2005. p. 1280.

9. Loeser JD. Economic implications of pain management. Acta Anaesthesiol Scand 1999;43(9):957-9.

10. Diacov N, Lima JRS. Absenteísmo odontológico. Rev Odont UNESP 1988;17(1/2):183-9.

11. Brasil. Ministério da Previdência Social. Auxilio Doença. Disponível em: <http://www.mpas.gov.br/conteudoDinamico.php?id=21>. Acesso em: 9 mar. 2010.

12. Brasil. Ministério da Previdência Social. Anuário Estatístico da Previdência Social 2009. Disponível em: <http://www.mpas.gov.br/conteudoDinamico. php?id=977>. Acesso em: 9 mar. 2010.

13. Elliott AM, Smith BH, Penny KI, et al. The epidemiology of chronic pain in the community. Lancet 1999;354(9186):1248-52.

14. Silva MC, Fassa AG, Valle NC. Chronic low back pain in a Southern Brazilian adult population: prevalence and associated factors. Cad Saude Publica 2004;20(2):377-85.

15. Moura AAG, Carvalho EF, Silva NJC. Repercussão das doenças crônicas não-transmissíveis na concessão de benefícios pela previdência social. Cienc Saúde Coletiva 2007;12(6):1661-72.

16. Picavet HS, Schouten JS. Musculoskeletal pain in the Netherlands: prevalences, consequences and risk groups, the DMC(3)-study. Pain 2003;102(1-2):167-78. 17. Waddell G. The Epidemiology of back pain. In: Waddell G, (editor). The back pain revolution. $2^{\text {nd }}$ ed. Churchill: Livingstone; 2004. p. 71-89.

18. Lee P, Helewa A, Goldsmith $\mathrm{CH}$, et al. Low back pain: prevalence and risk factors in an industrial setting. J Rheumatol 2001;28(2):346-51.

19. Sardá Júnior JA, Nicholas MK, Pimenta CAM, et al. Validação do questionário de incapacidade Roland Morris para dor em geral. Rev Dor 2010;11(1):28-36.

Apresentado em 07 de março de 2011.

Aceito para publicação em 05 de setembro de 2011. 